\title{
From Critical Problem to Interdisciplinary Application-Novel Material Development in Postgraduate Studies
}

\author{
Yixin Wang ${ }^{1}$, Hong Tao ${ }^{1, a, *}$ \\ School of Environment and Architecture, University of \\ Shanghai for Science and Technology, \\ Shanghai 200093, China \\ a taohong@usst.edu.cn;
}

\begin{abstract}
The postgraduate students majored in materials science and engineering for a master degree are often puzzled by where and how to start their scientific investigations. The hot problems in the applied disciplines can be exploited as the good start points for a series of innovations, which comprise fine teaching materials in higher education for master students both in theory and in practice. With one of the hottest problems in pharmaceutics, i.e. the dissolution of poorly water-soluble drug as a model, and with electrospinning as the applied nanotechnology, a new strategy was suggested in the present paper to demonstrate how to initiate the development of a new functional materials from the problems encountered in the applied subjects. The successful solution of the dissolution problem associated with the poorly water-soluble drug using the electrospun polymer nanofiber is just a pertinent example for explaining this strategy. Along this strategy, many other problems in a wide variety of applied fields can be similarly exploited as the start points to provoke the postgradaute students' interests and curiosities for initiating their scientific researches.
\end{abstract}

Keywords-Discipline-crossing innovation; Pharmaceutics; Material Science and Engineering; Electrospraying, Environment; Postgraduate student

\section{BACKGROUND ABOUT MATERIAL DESIGN}

Materials science and engineering is an interdisciplinary field, which involves the discovery and design of new materials [1]. The innovations of new materials design need, on one hand, the incorporated elements of physics, chemistry, and engineering. And on the other hand, these innovations should be useful in providing solutions to the problems in a wide variety of applied fields, such as energy, environments, biomaterials, and pharmaceutics. Particularly, many of the most pressing problems humans currently face are due to the

\author{
Di Wu², Yanglu Qu ${ }^{2}$, Yangchao Deng ${ }^{2}$, \\ Dengguang $\mathrm{Yu}^{2, \mathrm{~b}}$ \\ School of Material Science and Engineering, University of \\ Shanghai for Science and Technology, \\ Shanghai 200093, China \\ bydg017@usst.edu.cn
}

limits of the materials that are available. Thus, the needs in the applied fields are always the strong start point for developing new kinds of functional materials [2].

After many years' studies in their undergraduate stage, the postgraduate students majored in materials science and engineering for a master degree are keen to utilize their knowledge to develop new products. However, they are often puzzled about that where should be the start point and how to start a scientific project. This is mainly because of their limited knowledge about other fields. Thus, the hot problems from other disciplines can often be effective materials for provoking their interests and curiosities to begin their initial scientific investigations through an interdisciplinary manner.

Shown in Fig. 1 is a typical process for the interdisciplinary actions in designing new functional materials. The hot problems in the applied disciplines can be easily found from the literature review. The new materials can be designed to have the high functional performances for resolving the problems, which can be fulfilled through the reasonable selections of raw materials and the applied preparation methods. One of the hottest in pharmaceutics is the dissolution of poorly water-soluble drug, which is also a difficult issue in this area for over half a century. Today, more and more efforts have been paid to the amorphous nanomaterials for resolving this problem [3-7]. Based on these knowledge, the postgraduate students can begin their scientific investigations to take advantage of their professional knowledge. Taking considerations of the start materials (e.g. hydrophilic polymeric excipients and surfactant), a series of nanotechnologies such as electrospinning, electrospraying and others can be exploited to create new medicated biomaterials [4-13]. 


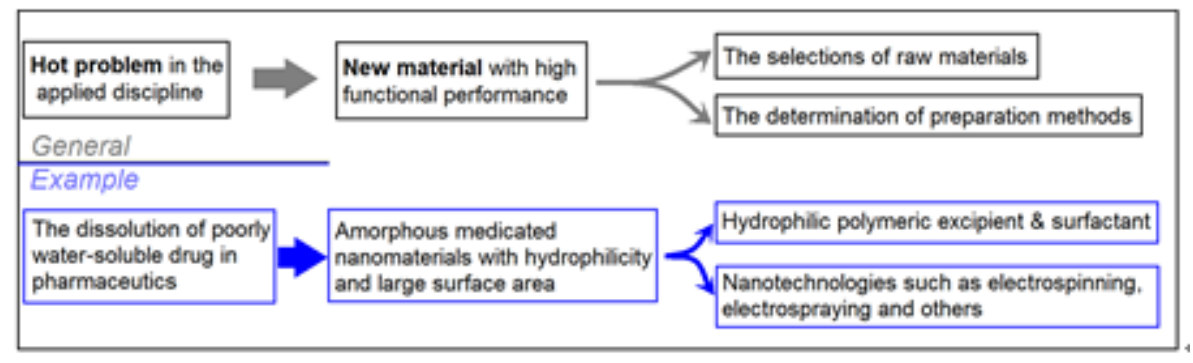

Fig. 1. The hot problem in an applied discipline can be utilized as a good start point for innovations, which can be further exploited as teaching materials.

The hot problem in an applied discipline can be utilized as a good start point for a series of innovations, which can be further exploited as teaching materials for the postgraduate education. One of the hottest problems in pharmaceutics is the dissolution of poorly water-soluble drug, which is utilized as an example of this investigation.

\section{ONE OF THE HOTTEST SPOT PROBLEMS IN PHARMACEUTICS}

During the past several decades, the numbers of poorly water-soluble drugs are always increasing with the progress of organic chemistry and bio-medical engineering, which comprises one of the hottest spot problems in the fields of pharmaceutics and also biomaterials [14]. Correspondingly, more and more methods are introduced into this field for effective delivery of these drugs [15]. A simple statistics based on Web of Science about the increase trend of poorly water-soluble drugs is shown in Fig. 1. The publication numbers were achieved by searching with "insoluble drug" or "poorly water-soluble drug" as "topic" within each five years from 1960. The sharp increase clearly demonstrates that the dissolution enhancement of poorly water-soluble drugs is one of the most difficult and intractable problems, which has drawn substantial attention simultaneously.

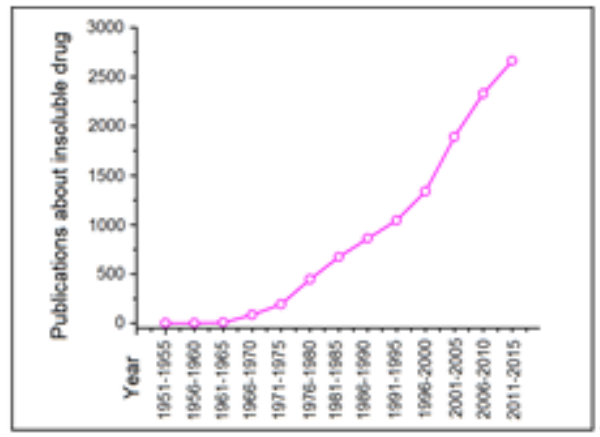

Fig. 2. The publications about poorly water-soluble drugs are rising.

The dissolution of poorly water-soluble drug comprises one of the hottest spot problems in pharmaceutics, which can be reflected from the never-stopping fast increase of publications about them. The data were achieved with "insoluble drug" as "topic" to search in Web of Science within in each five years.

\section{ELECTROSPINNING - ONE OF THE MOST POPULAR NANOTECHNOLOGIES}

Electrospinning, electrospraying and e-jetting printing are commonly termed as electrohydrodynamic atomization (EHDA) techniques, which are "top-down" processes for nano fabrications [16]. Different from traditional nano fabrication methods, electro-static energy is directly exploited to remove organic solvents from the working fluids for generating solid products during the EHDA processes [17]. These advanced material processing processes are very popular with potential applications of their products in a wide range of fields including pharmaceutics, ceramics, cosmetics energy, and environmental science and food industries.

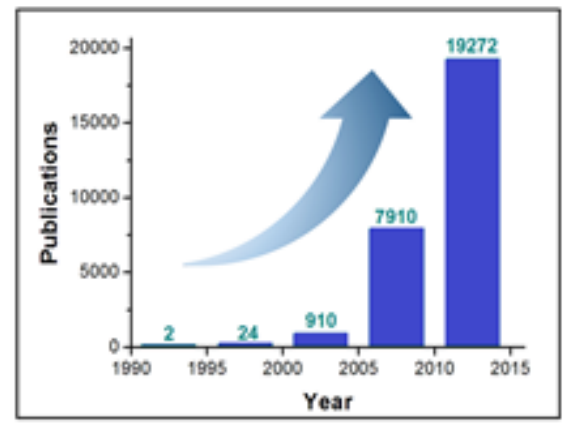

Fig. 3. The publications about electrospinning increase very fast.

The popularity of this technology should have a close relationship with the following three aspects. First, its great capability in generating nanostructures using a simple process. The past two decades has witnessed the great developments of electrospinning in manipulating the working fluids, which has evolved from the common single-fluid process, to double-fluid processes, and to multiple-fluid processes [18-22]. These techniques permit the creation of new types of sophisticated nanofibers with well-defined microstructures, novel morphologies, and/or new functions [23-26]. Second, it is very easy for implementation, a simple and cheap system with only one-step and straight forward generation process $[27,28]$. Third and also the most important is that the super functional performances of the electrospun nanofibers resulted from their unique physico-chemical properties such as smaller diameter, huger surface area and larger porosity. Meanwhile, the functions are easy to endowed and tailored through manipulations of the working processes. A simple search in Web of Science obviously demonstrates this trend, only 2 publications between 1990 and 1995 highly soared to 
near 20,000 publications between 2010 and 2015, meaning over 10 publication each day during the past five years. Electrospinning, as an advanced nanotechnology, has drawn more and more attentions during the past over twenty years, which can be utilized as a useful tool for resolving the problem of poorly water-soluble drugs. The data were achieved with "electrospinning" as "topic" to search in Web of Science within in each five years.

\section{RESOLUTION PROVIDED BY THE ADVANCED TECHNOLOGY FOR RESOLVING THE APPLIED PROBLEM}

Start from the hottest problem in pharmaceutics about poorly water-soluble drug and combination with the application of electrospinning, an interdisciplinary approach can be conceived for resolving this problem, i.e. to prepare amorphous hydrophilic nanomaterials for those insoluble drugs. "Amorphous hydrophilic nanomaterials" means three ways to work together to resolve this problems. First, the physical state is "amorphous", not crystalline, which is not easy to dissolve in water because that the high crystalline lattice energy must be overcome. Second, "hydrophilic" means the materials are not hydrophobic, i.e. the liquid-solid surface tensions are very small for the dissolution and diffusion of solute molecules to go into the bulk solutions. Third, "nanomaterials" means that there are a huge surface area for the drug molecules to contact the water and the later dissolution and diffusion.

Fig. 4 gives a full record about the preparation, characterization and functional applications of these amorphous hydrophilic nanomaterials. These nanomaterials are essentially nanocomposites, which were prepared using a modified coaxial electrospinning process (Fig. 5a, its upper-right inset is a digital image of the compound Taylor cone). Fig. 5b clearly demonstrated that these nanofibers had a smaller diameter within 1 micron and an obvious core-shell nanostructures (the upper-left inset). Fig. 5c verified that these nanofibers had the desired functional performnces, i.e. the fast dissolution of a poorly water-soluble drug, from 1 to 10 , the dissolution processes were finished within 1 minute.
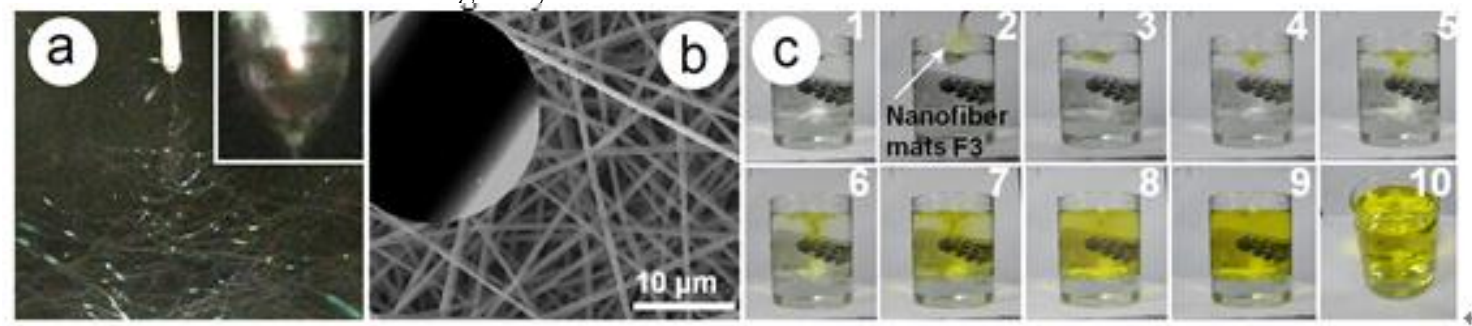

Fig. 4. The preparation of medicated nanofibers using coaxial electrospinning (a), their scanning electron microscopic images (b) and transmission electron microscopic image (the inset), and the fast dissolution of a poorly water-soluble drug (from 1 to 10 , the dissolution processes were finished within 1 minute) [15].

\section{STRATEGIES FOR NEW MATERIAL DESIGN BASED ON} INTERDISCIPLINARY STUDIES

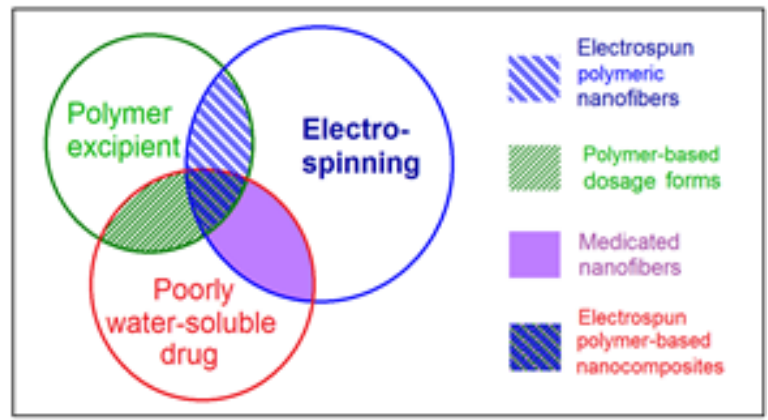

Fig. 5. The crossing-discipline innovations resulted from the requirement of pharmaceutics, which should be excellent teaching materials for postgraduate students

Based on the above-mentioned processes, the strategies for designing new types of functional nanomaterials can be concluded as follows. First, the needs or hot problems in the applied disciplines often comprise excellent start places to begin a new investigation or project for the postgraduate students, here is the dissolution of poorly water-soluble drug (Fig. 5). Second, the knowledge about the raw material systems and the reasonable selections for resolving the problem or meeting the needs are important. Third, the applications of advanced technologies in creating the designed new materials for realizing the desired functional performances should be carefully taken into considerations. Thus, there is an overlapped region in Fig. 5, which reflects the cross-disciplinary innovations among the related subjects including at least pharmaceutics, polymer science, biomaterials and electrohydrodynamic atomization.

\section{SUMMARY}

The present job successfully demonstrated a new strategy, which is very useful for initiating the postgraduate students' scientific investigations or projects. The needs or hot problems in other applied disciplines often comprise a fine strat point for the students to design new kinds of functional materials, which will finally meet the need or provide solutions to the problems. The dissolution enhancement of poorly water-soluble drug, as one of the hottest problems in pharmaceutics, was exploited as a beginning for conducting electrospinning to create new biomaterials, i.e. the electrospun amorphous hydrophilic nanomaterials. The positive results demonstrated that a fine start point, combined with an interdisciplinary overlap, can generate new fruits for new materials design and development. These strategies are particularly precious to the postgraduate students who are beginning with their initial scientific research learning. 
Along this strategy, many other problems in a wide variety of applied fields can be similarly exploited as the start points to provoke the postgradaute students' interests and curiosities for initiating their scientific researches. For example, we can apply electrospinning technology to the effluent from pharmaceuticals, which is not only a matter of urgency in the world of environmental protection, but also closely related to our lives. We will get the environmental problems and materials science and engineering together. So postgraduate students in the field of cross-disciplinary access to comprehensive knowledge, training disciplines cross the thinking of innovation, the theory and application of a seamless docking.

\section{ACKNOWLEDGEMENTS}

The financial supports from the following projects are appreciated: the National Natural Science Foundation of China (No. 51373101), the Shanghai Education Science Research Project (C17058), the 2016 key project on teachers teaching development in USST (CFTD17017Z) and the College Student Innovation Project of USST (Nos. XJ2017282, SH2017189, SH2017190 and SH2016191).

\section{REFERENCES}

[1] Anonymity, https://en.wikipedia.org/wiki/Materials_science, 21-March-2017.

[2] D.G. Yu, D. Pan, X. Wang, J. Luo, GR Williams, Cultivating undergraduate student's creativity by sharing scientific research experiences of academic staff. J. University of Shanghai for Science and Technology, 38 (2016) 179-183.

[3] Y.H. Wu, D.G. Yu, J.J. Li, Q. Wang, H.P. Li and X.Y. Li, Medicated multiple-component polymeric nanocomposites fabricated using electrospraying, Polym. Polym. Compos. 25 (2017) 57-62.

[4] Z.P. Liu, L. Cui, D.G. Yu, Z.X. Zhao and L. Chen. Electrosprayed core-shell solid dispersions of acyclovir fabricated using an epoxy-coated concentric spray head. Int. J. Nanomed., 9 (2014) 1967-19775.

[5] J. Yan, Y.H. Wu, D.G. Yu, G.R. Williams, S.M. Huang, W. Tao and J.Y. Sun, Electrospun acid-base pair solid dispersions of quercetin, RSC Adv., 4 (2014), 58265-58271.

[6] M. Jin, D.G. Yu, C.F.G.C. Geraldes, G.R. Williams, S.W.Annie Bligh, Theranostic fibers for simultaneous imaging and drug delivery, Mol. Pharm., 13 (2016) 2457-2465.

[7] D.G. Yu, K. White, N. Chatterton, Y. Li, L. Li, X. Wang, Structural lipid nanoparticles self-assembled from electrospun core-shell polymeric nanocomposites, RSC Adv., 5 (2015) 9462-9466.

[8] D.G. Yu, X.Y. Li, W. Chian, Y. Li and X. Wang, Influence of sheath solvents on the quality of ethyl cellulose nanofibers from a modified coaxial electrospinning, Biomed. Mater. Eng., 24 (2014) 695-701.

[9] J. Xie, H. Mao, D.G. Yu, G.R. Williams and M. Jin, Highly stable coated polyvinylpyrrolidone nanofibers prepared using modified coaxial electrospinning, Fiber. Polym. 15 (2014) 78-83.

[10] J. Yan, K. White, D.G. Yu and X.Y. Zhao, Sustained release multiple-component cellulose acetate nanofibers fabricated using a modified coaxial electrospinning process, J. Mater. Sci., 49 (2014) 538-547.

[11] C. Li, Z.H. Wang and D.G. Yu, Higher quality quercetin sustained release ethyl cellulose nanofibers fabricated using a spinneret with a Teflon nozzle, Colloid. Surface. B, 114 (2014): 404-409.

[12] W. Qian, D.G. Yu, Y. Li, Y.Z. Liao, X. Wang and L. Wang, Dual drug release electrospun core-shell nanofibers with tunable dose in the second phase, Int. J. Mol. Sci., 15 (2014) 774-786.

[13] D.G. Yu, Y. Xu, Z. Li, L.P. Du, B.G. Zhao and X. Wang. Coaxial electrospinning with mixed solvents: From flat to round Eudragit L100 nanofibers for better colon-targeted sustained drug release profiles, J. Nanomater., 2014 (2014) 967295.

[14] L. Cui, Z.P. Liu, D.G. Yu, S.P. Zhang, S.W.A. Bligh, N. Zhao, Electrosprayed core-shell nanopartciles of PVP and shellac for furnishing biphasic controlled release of ferulic acid. Colloid. Polym. Sci., 292 (2014) 2089-2096.

[15] X.Y. Li, Y.C. Li, D.G. Yu, Y.Z. Liao, X. Wang, Fast disintegrating quercetin-loaded drug delivery systems fabricated using coaxial electrospinning, Int. J. Mol. Sci., 14 (2013) 21647-21659.

[16] C. Li, D.G. Yu, G.R. Williams and Z.H. Wang. Fast-dissolving core-shell composite microparticles of quercetin fabricated using a coaxial electrospray process, PLOS One, 9 (2014) e92106.

[17] D.G. Yu, G.R. Williams, X. Wang, X.K. Liu, H.L. Li, S.W.A. Bligh, Dual drug release nanocomposites prepared using a combination of electrospraying and electrospinning, RSC Adv., 3 (2013) 4652-4658

[18] H.F. Wen, C. Yang, D.G. Yu, X.Y. Li and D.F. Zhang, Electrospun zein nanoribbons for treatment of lead-contained wastewater, Chem. Eng. J. 290 (2016), 263-272.

[19] C. Yang, D.G. Yu, D. Pan, X.K. Liu, X. Wang, S.W. A. Bligh and G. R Williams, Electrospun $\mathrm{pH}$-sensitive core-shell polymer nanocomposites fabricated using a tri-axial processes, Acta Biomater. 35 (2016) 77-86

[20] G.Z. Yang, H.P. Li, J.H. Yang, J. Wan and D.G. Yu, Influence of working temperature on the formation of electrospun polymer nanofibers. Nanoscale Res. Lett., 12 (2017) 1-10.

[21] F. Xu, Y. Xu and D.G. Yu, Zero-order controlled release nanofiber fabricated using coaxial electrospinning with polymer dilute solution as a sheath fluid, J. University of Shanghai for Science and Technology, 37 (2015) 165-168

[22] D.G. Yu, J.J. Li, M. Zhang, G.R. Williams, High-quality Janus nanofibers prepared using three-fluid electrospinning, Chem. Commun., 2017, DOI: 10.1039/C7CC01661A.

[23] C. Li, Z.H. Wang, D.G. Yu and G.R. Williams, Tunable biphasic drug release from ethyl cellulose nanofibers fabricated using a modified coaxial electrospinning process, Nanoscale Res. Lett., 9 (2014) 258.

[24] D.G. Yu, C. Yang, M. Jin, G.R. Williams, H. Zou, X. Wang, S.W.A Bligh, Medicated Janus fibers fabricated using a Teflon-coated side-by-side spinneret, Colloid. Surface. B, 138 (2016) 110-116.

[25] D.G. Yu, X.Y. Li, X. Wang, J.H. Yang, S.W.A. Bligh, G.R. Williams, Nanofibers fabricated using triaxial electrospinning as zero order drug delivery systems, ACS Appl. Mater. Interfaces 7 (2015) 18891-18897.

[26] C. Sealy, New spin on drug-loaded composite fibers, Materials Today, 19 (2016) 374-374

[27] G.Z. Yang, J.J. Li, D.G. Yu, M.F. He, J.H. Yang and G.R. Williams, Nanosized sustained-release drug depots fabricated using modified tri-axial electrospinning, Acta Biomater., 53 (2017) 233-241.

[28] D.G. Yu, X.Y. Li, W. Chian, Y. Li, X. Wang, The influence of sheath solvents on the quality of ethyl cellulose nanofibers from a modified coaxial electrospinning, Bio-med. Mater. Eng., 24 (2014) 695-701 